Pacific Journal of Mathematics

ESTIMATES FOR PARTIAL SUMS OF CONTINUED FRACTION 


\title{
ESTIMATES FOR PARTIAL SUMS OF CONTINUED FRACTION PARTIAL QUOTIENTS
}

\author{
Harold G. Diamond and JefFrey D. VaAler
}

Metric-type estimates are given for a class of partial sums involving continued fraction partial quotients. These results extend a well known theorem of Khinchin and yield an almost-everywhere estimate for the quantity in the title.

1. Introduction. For $\alpha$ an irrational number in $(0,1)$ let

$$
\alpha=\frac{1}{a_{1}+\frac{1}{a_{2}+\frac{1}{\ddots}}}=\left\langle 0, a_{1}, a_{2}, \ldots\right\rangle
$$

be the representation of $\alpha$ as a regular continued fraction ([4, Ch. X], [5]). The numbers $a_{n}=a_{n}(\alpha)$ are called the partial quotients of $\alpha$.

A well-known theorem of Khinchin [5], [6] in the metric theory of continued fractions asserts that if $F$ is an arithmetic function satisfying $F(r) \ll r^{1 / 2-\delta}$ for some $\delta>0$ and if $S_{N}(F, \alpha):=F\left(a_{1}(\alpha)\right)$ $+\cdots+F\left(a_{N}(\alpha)\right)$ for each positive integer $N$, then

$$
\lim _{N \rightarrow \infty} \frac{1}{N} S_{N}(F, \alpha)=\frac{1}{\log 2} \sum_{r=1}^{\infty} F(r) \log \left\{1+\frac{1}{r(r+2)}\right\}
$$

holds for almost all $\alpha$ in $(0,1)$. This result has been extended by others ([2, $\S 4],[7$, Theorem 4]). In particular, we note that the Birkhoff Ergodic Theorem implies that (1) holds if its right-hand side is absolutely convergent.

Here we shall establish analogues of (1) for arithmetic functions $F$ which grow more rapidly than is allowed by Khinchin's theorem. In particular we shall consider the case $F(r)=I(r)=r$ and estimate

$$
S_{N}(I, \alpha)=a_{1}(\alpha)+\cdots+a_{N}(\alpha)
$$

Khinchin noted at the end of his book Continued Fractions [5] that $S_{N}(I, \alpha) / N$ could not have a finite limit for most values of $\alpha$. Indeed, for almost all $\alpha$ the inequality $a_{n}(\alpha)>n \log n$ holds for an infinite sequence of integers $n$ in consequence of the following result of Borel and Bernstein 
[4, Theorem 197], [5, Theorem 30], [1, Theorem 4.1]:

LEMMA 1. Let $\varphi(1), \varphi(2), \ldots$ be a sequence of positive numbers. For almost all $\alpha \in(0,1)$ the inequality $a_{n}(\alpha)>\varphi(n)$ has a finite number of solutions $n$ if and only if $\sum_{n=1}^{\infty} 1 / \varphi(n)<\infty$.

Khinchin showed in [6] that

$$
\left(b_{1}+\cdots+b_{N}\right) /(N \log N) \rightarrow 1 / \log 2
$$

in measure as $N \rightarrow \infty$, where

$$
b_{n}=b_{n}(\alpha)= \begin{cases}a_{n}, & \text { if } a_{n}<n(\log n)^{4 / 3} \\ 0, & \text { otherwise. }\end{cases}
$$

The limit (2) cannot hold a.e., since for almost all $\alpha \in(0,1)$ the inequality $b_{n}>n \log n \log \log n$ holds for an infinite sequence of integers $n$ by Lemma 1.

The obstacle to a.e. convergence, as we shall see, is the occurrence of a single large value of $a_{n}$. Here we shall establish an analogue of (1) by excluding at most one summand.

THEOREM 1. Suppose that $F$ is a positive valued arithmetic function satisfying the bound

$$
\left\{\sum_{j \leq N} F(j)^{2} / j^{2}\right\} /\left\{\sum_{j \leq N} F(j) / j^{2}\right\}^{2} \leq N(\log N)^{-3 / 2-\varepsilon}
$$

for some $\varepsilon>0$. Then for almost all $\alpha \in(0,1)$ and for all $N$ exceeding $a$ number $N_{0}(\alpha)$, we have

$$
\begin{aligned}
S_{N}(f, \alpha)= & (1+o(1)) \frac{N}{\log 2} \sum_{r \leq N} F(r) \log \left\{1+\frac{1}{r(r+2)}\right\} \\
& +\vartheta_{+} \max _{1 \leq n \leq N} F\left(a_{n}(\alpha)\right) .
\end{aligned}
$$

Here $0 \leq \vartheta_{+}=\vartheta_{+}(N, \alpha, F) \leq 1$.

If we take $F(r)=I(r)=r$ we obtain

Corollary 1. For almost all $\alpha \in(0,1)$ there exists a number $N_{0}=$ $N_{0}(\alpha)$ such that

$$
S_{N}(I, \alpha)=\frac{1+o(1)}{\log 2} N \log N+\vartheta_{+} \max _{1 \leq n \leq N} a_{n}(\alpha)
$$

holds for all $N \geq N_{0}$. 
An immediate consequence of Lemma 1 and Corollary 1 is Then

Corollary 2. Let $0<\varphi(1)<\varphi(2)<\cdots$ satisfy $\sum_{n=1}^{\infty} 1 / \varphi(n)<\infty$.

$$
S_{N}(I, \alpha)=\frac{1+o(1)}{\log 2} N \log N+\vartheta_{+} \varphi(N)
$$

holds for almost all $\alpha \in(0,1)$ and all $N \geq N_{0}(\alpha)$.

There are two main steps in the proof of the theorem. First, we show that for most $\alpha$ there can be at most one "large" $a_{n}(\alpha)$. Next we estimate the variance of a truncated form of $S_{N}$. The theorem follows easily from these estimates.

2. Auxiliary results. Let $[x]$ denote the integer part of a real number $x$ and let $\{x\}=x-[x]$ denote the fractional part. Define $T$ : $(0,1) \rightarrow[0,1)$ by $T x=\{1 / x\}$. Then the partial quotients of (an irrational number) $\alpha$ are given by the formulas

$$
a_{1}(\alpha)=[1 / \alpha], \quad a_{n+1}(\alpha)=a_{1}\left(T^{n} \alpha\right), \quad n \geq 1 .
$$

(Rational numbers have terminating continued fraction expansions and require slight alteration of the formulas. This is not needed here, since the rationals form a set of measure zero.)

The so called Gauss measure $\mu$ is defined on Borel subsets of $(0,1)$ by

$$
\mu(E)=\frac{1}{\log 2} \int_{t \in E} \frac{d t}{1+t} .
$$

This measure satisfies the invariance relation

$$
\mu\left(T^{-1} E\right)=\mu(E), \quad E \text { a Borel set. }
$$

Note that $\mu$ and Lebesgue measure have the same null sets.

For $r$ and $k_{1}, k_{2}, \ldots, k_{r}$ positive integers, set

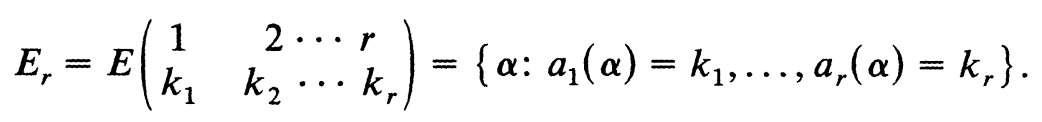

$E_{r}$ is called a fundamental interval of rank $r$. If $r$ and $s$ are positive integers, $B$ is any Borel set, and $E_{r}$ any fundamental interval of rank $r$, then $\mu$ satisfies the mixing relation [1, Chapter $1, \S 4$ ]

$$
\mu\left(E_{r} \cap T^{-r-s} B\right)=\mu\left(E_{r}\right) \mu(B)\left\{1+O\left(q^{s}\right)\right\}
$$

uniformly in $r, s, B$, and $E_{r}$. Here $q$ is some number in $(0,1)$. Together 
the preceding relations imply that

$$
\begin{aligned}
\mu & \left\{\alpha: a_{r}(\alpha)=m \text { and } a_{r+s}(\alpha)=n\right\} \\
& =\mu\left\{\alpha: a_{1}(\alpha)=m \text { and } a_{1+s}(\alpha)=n\right\} \\
& =\mu\left\{\left(\frac{1}{m+1}, \frac{1}{m}\right] \cap T^{-s-1}\left(\frac{1}{n+1}, \frac{1}{n}\right]\right\} \\
& =(\log 2)^{-2} \log \frac{(m+1)(m+1)}{m(m+2)} \log \frac{(n+1)(n+1)}{n(n+2)}\left\{1+O\left(q^{s}\right)\right\} .
\end{aligned}
$$

LEMMA 2. Let $c>1 / 2$, and for given $N \in \mathbf{Z}^{+}$set $N^{\prime}=N(\log N)^{c}$. For almost all $\alpha \in(0,1)$ there exist at most finitely many positive integers $N$ for which the inequalities

$$
a_{m}(\alpha)>N^{\prime}, \quad a_{n}(\alpha)>N^{\prime}
$$

hold for two distinct indices $m, n \leq N$.

Proof. Fix $m<n$. By a weak form of the mixing condition we have

$$
\begin{aligned}
& \mu\left\{\alpha \in(0,1): a_{m}(\alpha)>N^{\prime}, a_{n}(\alpha)>N^{\prime}\right\} \\
& \ll \mu\left\{\alpha: a_{m}(\alpha)>N^{\prime}\right\} \cdot \mu\left\{\alpha: a_{n}(\alpha)>N^{\prime}\right\} \\
&=\mu\left\{\alpha: a_{1}(\alpha)>N^{\prime}\right\}^{2} \ll\left(N^{\prime}\right)^{-2}=N^{-2}(\log N)^{-2 c} .
\end{aligned}
$$

It follows that the measure of the set on which (4) holds for some distinct indices $m, n \leq 2 N$ is of order at most $(\log N)^{-2 c}$. For $K=1,2, \ldots$ let

$$
U_{k}=\bigcup_{k \geq K}\left\{\alpha \in(0,1): a_{m}(\alpha)>\left(2^{k}\right)^{\prime}, a_{n}(\alpha)>\left(2^{k}\right)^{\prime}\right.
$$

$$
\text { for some distinct } \left.m, n \leq 2^{k+1}\right\} \text {. }
$$

Then

$$
\mu\left(U_{k}\right)<<\sum_{k \geq K} k^{-2 c} \rightarrow 0 \text { as } K \rightarrow \infty .
$$

For $\alpha \notin U_{k}$ and $N \geq 2^{K}$ there exists at most one index $n \leq N$ for which

$$
a_{n}(\alpha)>N(\log N)^{c} \text {. }
$$

3. Proof of the theorem. Given $\varepsilon>0$ and $N \in \mathbf{N}$, set

$$
a_{n}^{*}=a_{n, N}^{*}(\alpha)= \begin{cases}a_{n} & \text { if } a_{n} \leq N(\log N)^{1 / 2+\varepsilon / 4}=: N^{\prime} \\ 0 & \text { otherwise. }\end{cases}
$$


Define $F(0)=0$,

$$
\begin{aligned}
S_{N}^{*}(\alpha) & =S_{N}^{*}(F, \alpha)=\sum_{n \leq N} F\left(a_{n}^{*}(\alpha)\right), \\
J_{N} & =\int_{0}^{1} S_{N}^{*}(\alpha) d \mu(\alpha),
\end{aligned}
$$

and

$$
V_{N}=\int_{0}^{1}\left(S_{N}^{*}(\alpha)-J_{N}\right)^{2} d \mu(\alpha)
$$

We have

$$
\begin{aligned}
J_{N} & =\sum_{n=1}^{N} \int_{0}^{1} F\left(a_{n}^{*}(\alpha)\right) d \mu(\alpha)=N \int_{0}^{1} F\left(a_{1}^{*}(\alpha)\right) d \mu(\alpha) \\
& =N \sum_{j=1}^{N^{\prime}} F(j) \mu\left\{\alpha: a_{1}(\alpha)=j\right\} \\
& =\frac{N}{\log 2} \sum_{j=1}^{N^{\prime}} F(j) \log \left(1+\frac{1}{j(j+2)}\right) \asymp N \sum_{j=1}^{N^{\prime}} F(j) / j^{2} .
\end{aligned}
$$

(We say that $f \asymp g$ if $f \leq K_{1} g$ and $g \leq K_{2} f$ for suitable $K_{1}$ and $K_{2}$.)

Next we show that $V_{N} \ll N \sum_{j \leq N^{\prime}} F(j)^{2} / j^{2}$. We begin by writing

$$
\begin{aligned}
V_{N}+J_{N}^{2} & =\int_{0}^{1}\left|S_{N}^{*}(\alpha)\right|^{2} d \mu(\alpha) \\
& =\sum_{m, n=1}^{N} \int_{0}^{1} F\left(a_{m}^{*}(\alpha)\right) F\left(a_{n}^{*}(\alpha)\right) d \mu(\alpha)=\sum_{m, n=1}^{N} b_{m n},
\end{aligned}
$$

say. For $1>m<n \leq N$ we have

$$
\begin{aligned}
b_{m n} & =\sum_{j, k \leq N^{\prime}} F(j) F(k) \mu\left\{\alpha: a_{m}(\alpha)=j, a_{n}(\alpha)=k\right\} \\
& =\sum_{j, k \leq N^{\prime}} F(j) F(k) \mu\left(\frac{1}{j+1}, \frac{1}{j}\right] \mu\left(\frac{1}{k+1}, \frac{1}{k}\right]\left(1+O\left(q^{n-m}\right)\right) \\
& =J_{N}^{2} N^{-2}\left(1+O\left(q^{n-m}\right)\right) .
\end{aligned}
$$

The diagonal terms satisfy

$$
\begin{aligned}
b_{n n} & =\int_{0}^{1} F\left(a_{n}^{*}(\alpha)\right)^{2} d \mu(\alpha)=\int_{0}^{1} F\left(a_{1}^{*}(\alpha)\right)^{2} d \mu(\alpha) \\
& =\sum_{j \leq N^{\prime}} F(j)^{2} \mu\left\{\alpha: a_{1}(\alpha)=j\right\} \ll \sum_{j \leq N^{\prime}} F(j)^{2} / j^{2} .
\end{aligned}
$$


Thus we have

$$
\begin{aligned}
V_{N} & =\sum_{m, n=1}^{N} b_{m n}-J_{N}^{2} \ll J_{N}^{2} N^{-2} \sum_{m \leq n \leq N} q^{n-m}+N \sum_{j \leq N^{\prime}} F(j)^{2} / j^{2} \\
& \ll J_{N}^{2} / N+N \sum_{j \leq N^{\prime}} F(j)^{2} / j^{2} \\
& \ll N\left(\sum_{j \leq N^{\prime}} F(j) / j^{2}\right)^{2}+N \sum_{n \leq N^{\prime}} F(j)^{2} / j^{2} \\
& \ll N \sum_{j \leq N^{\prime}} F(j)^{2} / j^{2} .
\end{aligned}
$$

The last relation follows from the Cauchy-Schwarz inequality.

Now we apply the estimate of $V_{N}$ to show that

$$
S_{N}^{*}(\alpha)=(1+o(1)) J_{N}
$$

for most values of $\alpha$. Let

$$
c(k)=\left[\exp k^{1-\varepsilon / 4}\right], \quad k=1,2, \ldots
$$

We have

$$
\begin{gathered}
\int_{\alpha=0}^{1} \sum_{k=1}^{\infty}\left(S_{c(k)}^{*}(\alpha)-J_{c(k)}\right)^{2}\left(c(k) \sum_{j \leq c(k)^{\prime}} \frac{F(j)^{2}}{j^{2}} k^{1+\varepsilon / 4}\right)^{-1} d \mu(\alpha) \\
\ll \sum_{k=1}^{\infty} k^{-1-\varepsilon / 4}<\infty .
\end{gathered}
$$

It follows that the integrand in the last integral is finite a.e. and hence

$$
S_{c(k)}^{*}(\alpha)-J_{c(k)}=o\left\{c(k) \sum_{j \leq c(k)^{\prime}} \frac{F(j)^{2}}{j^{2}} k^{1+\varepsilon / 4}\right\}^{1 / 2}
$$

for almost all $\alpha$. The hypothesis of Theorem 1 and a small calculation show that

$$
c(k) \sum_{j \leq c(k)^{\prime}} \frac{F(j)^{2}}{j^{2}} k^{1+\varepsilon / 4} \ll J_{c(k)}^{2} /(\log c(k))^{\varepsilon / 12}=o\left(J_{c(k)}^{2}\right),
$$

provided that $\varepsilon<1$. Thus

$$
S_{c(k)}^{*}(\alpha)=(1+o(1)) J_{c(k)} \quad \text { a.e. }
$$

Suppose that $c(k-1)<N \leq c(k)$. Then

$$
S_{c(k-1)}^{*}(\alpha) \leq S_{N}^{*}(\alpha) \leq S_{c(k)}^{*}(\alpha) \text {, }
$$


and so, off a set of measure 0 ,

$$
(1+o(1)) J_{c(k-1)} \leq S_{N}^{*}(\alpha) \leq(1+o(1)) J_{c(k)} .
$$

Now we show that $J_{c(k)} \sim J_{c(k-1)}$. Recall that

$$
J_{N}=\frac{N}{\log 2} \sum_{j \leq N^{\prime}} F(j) \log \left(1+\frac{1}{j(j+2)}\right) .
$$

Another small calculation shows that

$$
c(k-1)=\left(1+O\left(k^{-\varepsilon / 4}\right)\right) c(k),
$$

so $c(k-1) \sim c(k)$ as $k \rightarrow \infty$. It remains to show that

$$
\sum_{j \leq c(k)^{\prime}} F(j) \log \left(1+\frac{1}{j(j+2)}\right) \sim \sum_{j \leq c(k-1)^{\prime}} F(j) \log \left(1+\frac{1}{j(j+2)}\right) .
$$

We shall show (5) and the final approximation of $S_{N}^{*}$ by using

LemMA 3. Let $F$ satisfy the hypotheses of Theorem 1. Then, as $N \rightarrow \infty$,

$$
\sum_{N<r \leq N \log N} \frac{F(r)}{r^{2}}=o\left(\sum_{r \leq N} \frac{F(r)}{r^{2}}\right) .
$$

Proof. The Cauchy Schwarz inequality and condition (3) yield

$$
\begin{aligned}
\sum_{N<r \leq N \log N} \frac{F(r)}{r^{2}} & \leq\left\{\sum_{r \leq N \log N} \frac{F(r)^{2}}{r^{2}}\right\}^{1 / 2}\left\{\sum_{r>N} \frac{1}{r^{2}}\right\}^{1 / 2} \\
& \leq \sum_{r \leq N \log N} \frac{F(r)}{r^{2}}(\log N)^{-1 / 4-\varepsilon / 2}
\end{aligned}
$$

Thus, as $N \rightarrow \infty$,

$$
(1-o(1)) \sum_{N<r \leq N \log N} \frac{F(r)}{r^{2}} \leq o(1) \sum_{r \leq N} \frac{F(r)}{r^{2}},
$$

and the lemma follows.

Returning to the proof of Theorem 1, we see first that (5) holds, and hence

$$
S_{N}^{*}(\alpha)=(1+o(1)) J_{N} \text { a.e. }
$$

The lemma also implies that

$$
S_{N}^{*}(\alpha)=(1+o(1)) \frac{N}{\log 2} \sum_{j \leq N} F(j) \log \left(1+\frac{1}{j(j+2)}\right) \quad \text { a.e. }
$$


since

$$
\sum_{N<j \leq N^{\prime}} F(j) \log \left(1+\frac{1}{j(j+2)}\right)
$$

is negligible.

Finally, we have by Lemma 2, for almost all $\alpha$ and all sufficiently large $N$,

$$
0 \leq S_{N}(\alpha)-S_{N}^{*}(\alpha) \leq F\left(\max _{1 \leq n \leq N} a_{n}(\alpha)\right) \leq \max _{1 \leq n \leq N} F\left(a_{n}(\alpha)\right) .
$$

This inequality and the last estimate of $S_{N}^{*}$ establish the theorem.

It would be interesting to learn whether Theorem 1 could be established by ergodic methods.

4. Further results. In this section we consider cases in which $S_{N}(I, \alpha)$ can be estimated by $N(\log N) /(\log 2)$ alone and when by $\varphi(N)$ alone, where $\sum_{n=1} 1 / \varphi(n)<\infty$.

First we note that for any $\varepsilon>0$ and fixed $N \in \mathbf{Z}^{+}$we have

$$
\mu\left\{\alpha \in(0,1):\left|\frac{S_{N}(I, \alpha)}{N \log N}-\frac{1}{\log 2}\right|>\varepsilon\right\} \ll \frac{1}{\varepsilon \log N} .
$$

(The implied constant here is absolute.)

This bound is achieved by setting

$$
a_{n}^{* *}=a_{n, N, \varepsilon}^{* *}(\alpha)= \begin{cases}a_{n}, & \text { if } a_{n}<\varepsilon N \log N==: N^{\prime \prime} \\ 0, & \text { otherwise. }\end{cases}
$$

We compute the variance of the sum function $S_{N}^{* *}$ as before and apply Chebyshev's estimate to obtain

$$
\mu\left\{\alpha:\left|S_{N}^{* *}(\alpha)-\frac{N \log N}{\log 2}\right|>\varepsilon N \log N\right\} \ll \frac{1}{\varepsilon \log N} .
$$

Also, for each $n \leq N$,

$$
\mu\left\{\alpha: a_{n}(\alpha)>\varepsilon N \log N\right\} \ll \frac{1}{\varepsilon N \log N},
$$

and estimate (6) follows.

Next, we show directly a sharp one sided estimate of Pruitt [9, Theorem 5.2].

Corollary 3. For $N \geq 3$ set $\beta(N)=\exp \left(k \log ^{2} k\right) k \log ^{2} k$ for

$$
\exp \left((k-1) \log ^{2}(k-1)\right)<N \leq \exp \left(k \log ^{2} k\right) \text {. }
$$


Then, for almost all $\alpha \in(0,1)$

$$
\limsup _{N \rightarrow \infty} \frac{S_{N}(I, \alpha)}{\beta(N)}=\frac{1}{\log 2} .
$$

Proof. In Corollary 2 set

$$
\varphi(N)=\beta(N) /(\log \log 10 k) .
$$

An easy calculation shows that $\sum 1 / \varphi(N)<\infty$. If $N$ satisfies (7), then by Corollary 2

$$
S_{N}(I, \alpha) \leq \frac{1+o(1)}{\log 2} \beta(N)+\beta(N) / \log \log 10 k \quad \text { a.e., }
$$

so $\lim \sup S_{N}(I, \alpha) / \beta(N) \leq 1 / \log 2$ a.e. On the sequence $N_{k}=$ $\exp \left(k \log ^{2} k\right)$, the ratio $S_{N}(I, \alpha) / \beta(N)$ converges to $1 / \log 2$ a.e.

In another direction, we show that in Corollary $2, \varphi(N)$ dominates $N \log N$ for "most" values of $N$.

LEMMA 4. Suppose that $0<\varphi(1) \leq \varphi(2) \leq \cdots$ and $\sum_{n=1}^{\infty} 1 / \varphi(n)<$ $\infty$. Let

$$
S=\left\{n \in \mathbf{Z}^{+}: \varphi(n)<n \log n\right\} .
$$

Then $S$ has logarithmic density zero.

Proof. Let $T=\left\{\nu \in \mathbf{Z}^{+}:\left(2^{\nu-1}, 2^{\nu}\right] \cap S \neq \varnothing\right\}$. Suppose $\nu \in T$. Then there exists an integer $n$ such that $2^{\nu-1}<n \leq 2^{\nu}$ and $\varphi(n)<n \log n$, so

$$
\sum_{n / 2<k \leq n} \frac{1}{\varphi(k)} \geq \frac{n-[n / 2]}{n \log n} \geq \frac{1}{2 \log n} \geq \frac{1}{2 \nu \log 2} .
$$

Thus

$$
\sum_{\nu \in T} \frac{1}{\nu} \leq 4(\log 2) \sum_{k=1}^{\infty} 1 / \varphi(k)<\infty .
$$

Also, we have

$$
\sum_{\substack{k \in S \\ 2^{\nu-1}<k \leq 2^{\nu}}} \frac{1}{k} \leq \begin{cases}\log 2, & \nu \in T \\ 0, & \nu \notin T .\end{cases}
$$

With $y=(\log x) / \log 2$ we have

$$
\begin{aligned}
& \delta_{x}:=\frac{1}{\log x} \sum_{\substack{k \leq x \\
k \in S}} \frac{1}{k} \leq \frac{1}{y \log 2} \sum_{\nu \leq y+1} \sum_{\substack{k \in S \\
2^{\nu-1}<k \leq 2^{\nu}}} \frac{1}{k} \\
& \leq \frac{1}{y} \sum_{\substack{\nu \leq y+1 \\
\nu \in T}} 1=\frac{T(y+1)}{y}, \text { say. }
\end{aligned}
$$


We have from (8) that

$$
\frac{1}{N}(T(N)-T(N / 2)) \leq \sum_{\substack{\nu \in T \\ n / 2<\nu \leq N}} \frac{1}{\nu} \rightarrow 0
$$

as $N \rightarrow \infty$. Thus $T(y)=o(y)$ as $y \rightarrow \infty$. Finally,

$$
\limsup _{x \rightarrow \infty} \delta_{x} \leq \limsup _{y \rightarrow \infty} \frac{T(y+1)}{y}=0 .
$$

COROLlary 4. Suppose that $\varphi$ satisfies the hypotheses of Lemma 4. Then for almost all $\alpha \in(0,1)$

$$
S_{N}(I, \alpha) \ll \varphi(N)
$$

holds for all integers $N$ outside a set of logarithmic density zero.

Acknowledgment. We are indebted to Professor Walter Philipp for several helpful conversations.

\section{REFERENCES}

[1] P. Billingsley, Ergodic Theory and Information, Wiley, New York, 1965.

[2] W. Doeblin, Remarques sur la théorie métrique des fractions continues, Compositio Math., 7 (1940), 353-371.

[3] P. Erdös, Some remarks on diophantine approximation, J. Indian Math. Soc., 12 (1948), 67-74.

[4] G. H. Hardy and E. M. Wright, An Introduction to the Theory of Numbers, 5th Edition, Clarendon Press, Oxford, 1979.

[5] A. Ya. Khinchin, Continued Fractions, U. Chicago Press, Chicago, 1964.

[6] _ Metrische Kettenbruchprobleme, Compositio Math., 1 (1935), 361-382.

[7] W. Phillip, Some metrical theorems in number theory, Pacific J. Math., 20 (1967), 109-127.

[8] __ Some metrical theorems in number theory, II, Duke Math. J., 38 (1970), 447-458.

[9] W. E. Pruitt, General one-sided laws of the iterated logarithm, Ann. of Probability, 9 (1981), 1-48.

Received August 28, 1984 and in revised form January 22, 1985. Research supported in part by grants from the National Science Foundation.

UNIVERSITY OF ILLINOIS

URBANA, IL 61801

AND

ThE UNIVERSITY OF TEXAS

Austin, TX 78712 


\section{PACIFIC JOURNAL OF MATHEMATICS EDITORS}

\author{
V. S. VARADARAJAN (Managing Editor) \\ University of California \\ Los Angeles, CA 90024 \\ Hebert Clemens \\ University of Utah \\ Salt Lake City, UT 84112 \\ Charles R. DePrima \\ California Institute of Technology \\ Pasadena, CA 91125
}

R. FINN

Stanford University

Stanford, CA 94305

HermanN FlaschKa

University of Arizona

Tucson, AZ 85721

RAMESH A. GANGOLlI

University of Washington

Seattle, WA 98195

ROBION KIRBY

University of California

Berkeley, CA 94720

ASSOCIATE EDITORS
R. ARENS
E. F. BECKENBACH
B. H. NEUMANN
F. WOLF
K. YoshidA (1906-1982)

C. C. Moore

University of California

Berkeley, CA 94720

H. SAMELSON

Stanford University

Stanford, CA 94305

HAROLD STARK

University of California, San Diego

La Jolla, CA 92093

\section{SUPPORTING INSTITUTIONS}

UNIVERSITY OF ARIZONA

UNIVERSITY OF BRITISH COLUMBIA

CALIFORNIA INSTITUTE OF TECHNOLOGY

UNIVERSITY OF CALIFORNIA

MONTANA STATE UNIVERSITY

UNIVERSITY OF NEVADA, RENO

NEW MEXICO STATE UNIVERSITY

OREGON STATE UNIVERSITY
UNIVERSITY OF OREGON

UNIVERSITY OF SOUTHERN CALIFORNIA

STANFORD UNIVERSITY

UNIVERSITY OF HAWAII

UNIVERSITY OF TOKYO

UNIVERSITY OF UTAH

WASHINGTON STATE UNIVERSITY

UNIVERSITY OF WASHINGTON 


\section{Pacific Journal of Mathematics}

Vol. 122, No. $1 \quad$ January, 1986

Michael James Cambern, Near isometries of Bochner $L^{1}$ and $L^{\infty}$ spaces ....1 Kun Soo Chang, Gerald William Johnson and David Lee Skoug, The

Feynman integral of quadratic potentials depending on two time

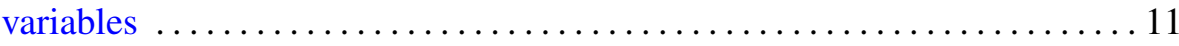

Robert Coleman, One-dimensional algebraic formal groups $\ldots \ldots \ldots \ldots \ldots 35$

Alberto Collino, The Abel-Jacobi isomorphism for the cubic fivefold .......43

N. J. Dev and S. S. Khare, Finite group action and vanishing of $N_{*}^{G}[F] \ldots 57$

Harold George Diamond and Jeffrey D. Vaaler, Estimates for partial sums

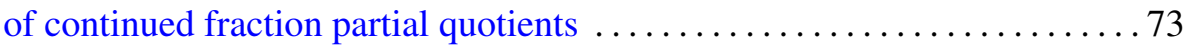

Kenneth R. Goodearl, Patch-continuity of normalized ranks of modules

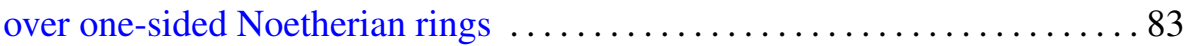

Dean Robert Hickerson and Sherman K. Stein, Abelian groups and

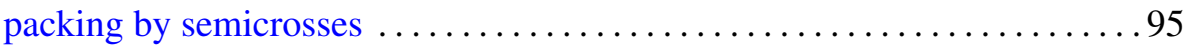

Karsten Johnsen and Harmut Laue, Fitting structures $\ldots \ldots \ldots \ldots \ldots 11$

Darren Long, Discs in compression bodies . ................... 129

Joseph B. Miles, On the growth of meromorphic functions with radially distributed zeros and poles ........................... 147

Walter Volodymyr Petryshyn, Solvability of various boundary value problems for the equation $x^{\prime \prime}=f\left(t, x, x^{\prime}, x^{\prime \prime}\right)-y \ldots \ldots \ldots \ldots \ldots . \ldots 169$

Elżbieta Pol, The Baire-category method in some compact extension problems

Masami Sakai, A new class of isocompact spaces and related results 211

Thomas Richard Shemanske, Representations of ternary quadratic forms and the class number of imaginary quadratic fields ..

Tsuyoshi Uehara, On class numbers of cyclic quartic fields 